\title{
Using Assistive Technologies in Autism Care Centers to Support Children Develop Communication and Language Skills. A Case Study: Albania
}

\author{
Ardiana Sula \\ Polytechnic University of Tirana, Faculty of Information Technology \\ Mother Teresa Square, Nr. 4, Tirana, Albania \\ E-mail: ardianasula@gmail.com,
}

Evjola Spaho

Graduate School of Engineering, Fukuoka Institute of Technology (FIT) 3-30-1 Wajiro-Higashi, Higashi-Ku, Fukuoka 811-0295, Japan

\section{Doi:10.5901/ajis.2014.v3n1p203}

\section{Abstract}

In this paper, we propose the use of the smart environment based on Internet of Things (IoT) and P2P technology as a form of assistive environment in autism care centers to support children develop communication and language skills. We are going to propose the use of the smart environment in Albanian Regional Center for Autism in Tirana, Albania. The smart environment is proposed as an assistive environment for this center in order to support students' learning during the time that they come to the center for support. Our proposed system uses JXTA-Overlay platform and SmartBox device to monitor the children and create P2P communication between children, caregivers and therapists. Various visual systems, such as objects, photographs, realistic drawings, line drawings, and written words used with assorted modes of technology can support engaged learning and the can increase and maintain the concentration of children with ASD during learning.

Keywords: P2P technology, Internet of Things, Smart environment, SmartBox, JXTA-Overlay, RFID, Assistive Technologies, Autism Spectrum Disorder.

\section{Introduction}

Many children with autism are highly interested and motivated by smart devices such as computers and touch screen tablets. By using these types of assistive technology devices children with autism can interact, make choices, respond, gain new skills and become more independent.

The combination of the smart environment with different assistive technologies can be used in schools and other centers to support students with autism overcome communication barriers and develop new language skills.

Most of the socially assistive research to date is focused on Autism Spectrum Disorder (ASD). Autism is a neurological disorder that affects the ability to communicate and interact socially. One way to cope with this problem is using assistive technologies and finding ways how to benefit from the use of technology to help these children. Individuals with cognitive disabilities, developmental and social disorders constitute another growing population that may benefit from assistive applications in the contexts of special education, therapy, and training.

The cause of the increasing number of children with autism is not yet known. However, many researchers agree that early intervention is critical to enabling a positive long-term outcome. Even with early intervention, many individuals will need high levels of support and care throughout their lives.

An assistive smart environment has the potential to enhance the quality of life for broad populations of users: elderly, individuals with physical impairments and those in rehabilitation therapy, and individuals with cognitive disabilities and developmental and social disorders [1].

The advance of the technology is leading to smart objects being capable of identifying, locating, sensing and connecting and thus leading to new forms of communication between people and things; and, things themselves. Nowadays, the researchers and developers are focused on developing various smart devices that allow users to control and monitor events in consumer-based appliances, home electronics, and home security systems. These devices vary from low tech to high tech devices. As these devices become more common, the need will increase for a home 
networking strategy that will allow all data, voice, and smart devices to be accessed at anytime from anywhere.

Internet of Things (IOT) will be a key part of the future Internet, and Radio Frequency Identification (RFID) tags, allow objects to be uniquely identified, to determine the location, to sense changes in physical data and to connect and communicate with a corresponding transponder. Children with autism are the subjects of the research presented here, because there is no current cure for autism and the primary care goal is in creating a supportive environment.

ASDs affect one out of every 88 children in the U.S. They occur more often among boys than girls [2].

There are no studies in Albania focused on the identification of the number of children with autism, but according to the specialists of the center there is a high possibility that the number of children with autism in Albania is the same with those of other countries [3].

In this paper, we propose the use of a system based on an assistive smart environment for supporting learning and improving the quality of life of children in autism support centers. Our proposed system includes the use the computer, sensors, RFID tag reader and SmarBox device. The proposed system provides to children assistive learning by using tagged cards during activities as picture learning, matching identical objects, matching similar objects, sorting into categories, working with people and emotions categories and basic math category. Our proposed system doesn't intend to substitute face to-face interaction, but rather provide learning activities for students with autism as a form of differentiated instruction. The system will provide a learning environment which is predictable, concrete, self-paced, and promotes better visual information processing. The actively engagement of students with ASD in this learning environment can increase attention and decrease anxiety related behaviors [4].

The rest of the paper is organized as follows. In Section II, we give an overview of the various current networking technologies. In Section 3 we present the related work on the smart environment. In Section IV, we propose the use of the smart environment in the Regional Center for Autism in Tirana as a medical application aiming to assist/support children with ASD getting new skills.

We present conclusions and future work in Section V.

\section{Related Work on Various Networking Technologies and Various Assistive Technologies}

Advances in data collection technology, such as embedded devices and RFID technology have led to an increasing number of smart devices that allow users to control and monitor events in consumer-based appliances, home electronics, and home-security systems. As these devices become more common, the need will increase for a home networking strategy that will allow all data, voice, and smart devices to be accessed at anytime from anywhere [5].

Home networking is the collection of elements that process, manage, transport, and store information, enabling the connection and integration of multiple computing, control, monitoring, and communication devices in the home.

Robots are being steadily introduced into modern everyday life and are expected to play a key role in the near future. Assistive robotics in general and socially assistive robotics in particular have the potential to enhance the quality of life for broad populations of users: the elderly, individuals with physical impairments and those in rehabilitation therapy, and individuals with cognitive disabilities and developmental and social disorders [6].

Peer to Peer (P2P) systems appeared as a new paradigm after client-server and became quite popular for filesharing among Internet users. Such systems benefit from high scalability and fault tolerance. Today research is focused on the design, implementation and deployment of full featured P2P networks that integrate end-devices. P2P is a very good approach to an efficient platform for e-learning and robot control.

A Wireless Personal Area Network (WPAN) is a low range wireless network which covers an area of only a few dozen meters. This sort of network is generally used for linking peripheral devices (like printers, cellphones, tablets and home appliances) or a Personal Digital Assistant (PDA) to a computer, or just two nearby computers, without using a hard-wired connection. Wireless Sensor Networks (WSNs) are increasingly gaining impact in our daily lives. They are finding a wide range of applications in various domains, including healthcare, assisted and enhanced-living scenarios, industrial and production monitoring, control networks, and many other fields.

\subsection{Internet of Things (loT)}

The term IoT has recently become popular to emphasize the vision of a global infrastructure of networked physical objects. IoT is a new type of Internet application which enables the things/objects in our environment to be active participants with other members of the network, by sharing their information on a global scale using the same Internet Protocol (IP) that connects to the Internet. The descriptive models for Internet of Things are introduced based on two 
attributes ("being an Internet", "relating to thing's information") and four different features (only for thing's information, coded by UID or EPC, stored in RFID electronic tag, uploaded by non-contact reading with RFID reader) [7].

The IOT creates human-machine or machine-to-machine communications. In this way the things/objects are capable of recognizing events and changes in their surroundings and are acting and reacting autonomously largely without human intervention in an appropriate way. The major objectives for loT applications and services are the creation of smart environments/spaces and self-aware things for smart transport, products, cities, buildings, energy, health, social interaction and living applications (see Fig. 1).

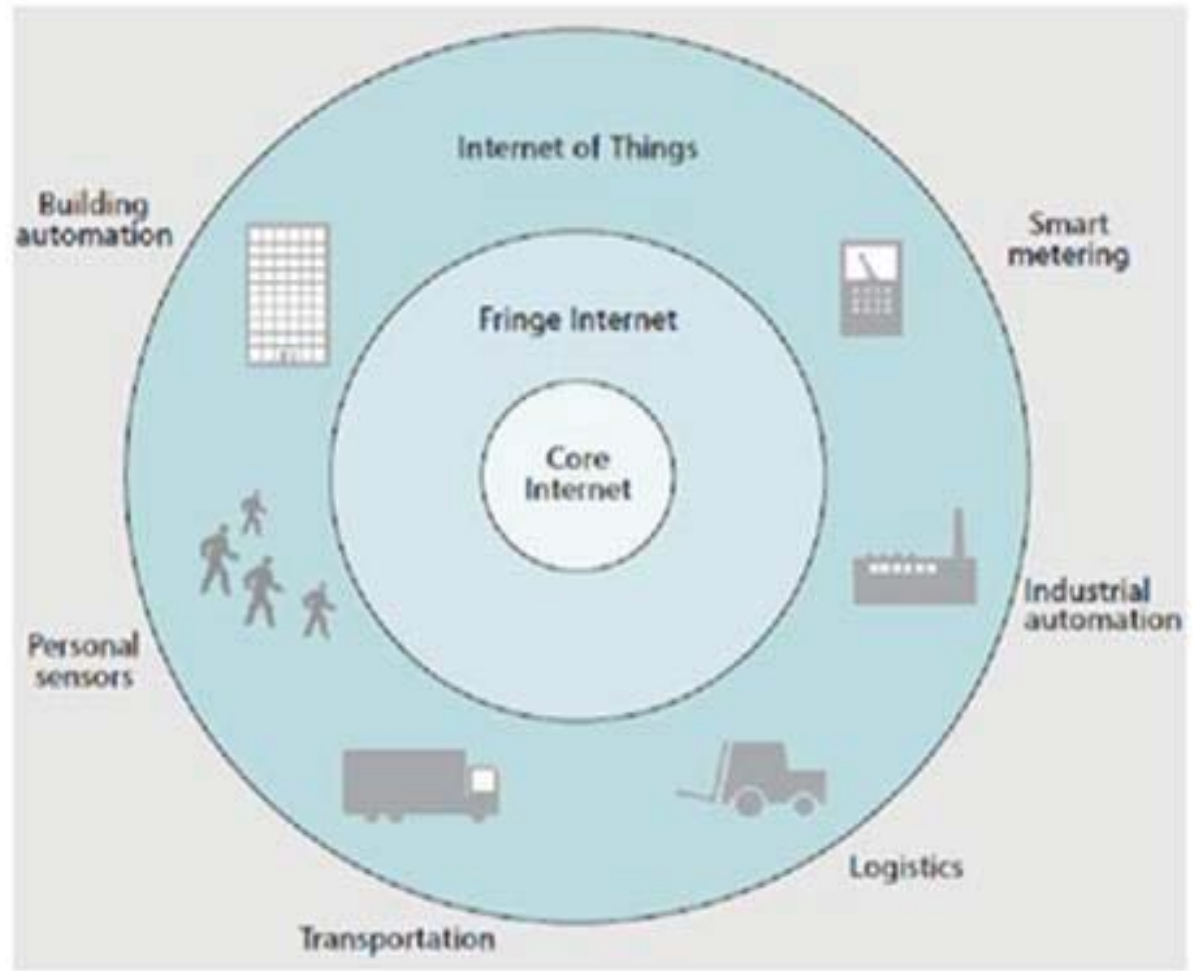

Figure 1: IoT model.

IOT development depends on successful development of RFID technology and design of various new applications and business models. RFID and sensor network technologies will rise to meet this new challenge, in which information and communication systems are embedded in the environment. RFID is primarily used to identify objects from a distance of a few meters, with a stationary reader typically communicating wirelessly and can be used to determine the approximate location of objects provided the position of the reader is known. RFID systems consist of three main components: RFID tags, the RFID readers, and RFID software. The IoT will accelerate and expand, since the IPv6 (Internet Protocol version 6) implementation supports tremendous growth of IP addresses, giving broader ability for every physical thing to connect to the Internet and communicate with any other object.

\section{The Use of the Smart Environment Based on Internet of Things (IoT) and P2P Technology for Supporting Children with ASD}

Assistive Technologies (ATs) such as Ambient Assisted Living (AAL) encompasses technical systems to support children with autism in their daily routine to allow an independent and safe lifestyle and an assistive learning environment. The combination of smart technologies results in an applied loT infrastructure for AAL scenarios. A central AAL paradigm can be realized through the IoT, where the children with autism live in their homes with smart objects, thus smart homes, 
communicating to the outside world in an intelligent and goal-orientated manner.

We implemented in our previous work the combination of IOT, P2P, Web and sensor technologies for monitoring, checking, controlling the health situation and assisting children with ASD. Our implemented loT based system is shown in Fig. 4. Our system allows sending information about the children state in real time to therapists using P2P technology and also allows children to interact with other children and parents. Taking advantages of the fact that students with autism are good at using technology, we proposed and implemented a new e-learning system in order to increase the children's concentration by combining computer, projector, SmartBox and sensors.

A study presented in 2008 by Temple University researchers found out that helping children adjust to different sensations led to fewer autistic mannerisms and better behavior [8]. Our system supports figures, sounds, music, videos, tags and colorful presentations which promise to increase the interaction with smart environment and children's concentration during a learning task. Using sensor technologies, the body movement is measured and all the data are saved in a data base. One of the learning barriers for some of the children with ASD is the short attention span for most lessons. We are going to propose the use of this smart environment system in order to create a suitable environment for children during their learning.

\subsection{JXTA-Overlay}

P2P communication between children, caregivers and therapists can be build using JXTA-Overlay Platform. JXTA Overlay project is an effort to use JXTA technology for building an overlay on top of JXTA offering a set of basic primitives (functionalities) that are most commonly needed in JXTA-based applications. The proposed overlay comprises the following primitives: peer discovery, peer's resources discovery, resource allocation, task submission and execution, file/data sharing, discovery and transmission, instant communication, peer group functionalities (groups, rooms etc.), monitoring of peers, groups and tasks. The overlay is built on top of JXTA layer and provides a set of primitives that can be used by other applications, which on their hand, will be built on top of the overlay, with complete independence.

The most important part in a P2P system is the communication between peers. By using JXTA-Overlay, it is possible to overcome, firewalls, routers, NATs, and bridges in the private networks.

\subsection{SmartBox}

In the previous implemented system the use of SmartBox has shown positive effects by increasing the learner's motivation and concentration during online learning. The research has shown that many students with autism have social interaction challenges that are very difficult to address outside real social situations. Computer use can provide students with a sense of control and consistency and students can use different educational software's to practice communication skills in a lower stress environment compared to real environment. In our previous work, we implemented the smart environment based on P2P technology in order to get and maintain the child's attention by obtaining the calm-alert state window in which our ability to function is maximized [9]. In this state the child's nervous system is sufficiently aroused for peak attention and task performance which enhances a person's ability to register and orient to sensory information. The SmartBox device is integrated with our system as a useful tool for monitoring and controlling children activities. The size of the SmartBox is $35 \times 7 \times 12 \mathrm{~cm}$ (see Fig. 3). The SmartBox has the following sensors and functions:

- Body Sensor for detecting body and hand movement.

- Chair or Bed Vibrator Control for vibrating the chair or bed.

- Light Control for adjusting the room light.

- Smell Control for controlling the room smell.

- Sound Control to emit relaxing sounds.

- Remote Control Socket for controllingAC 100V socket (on-off control). 


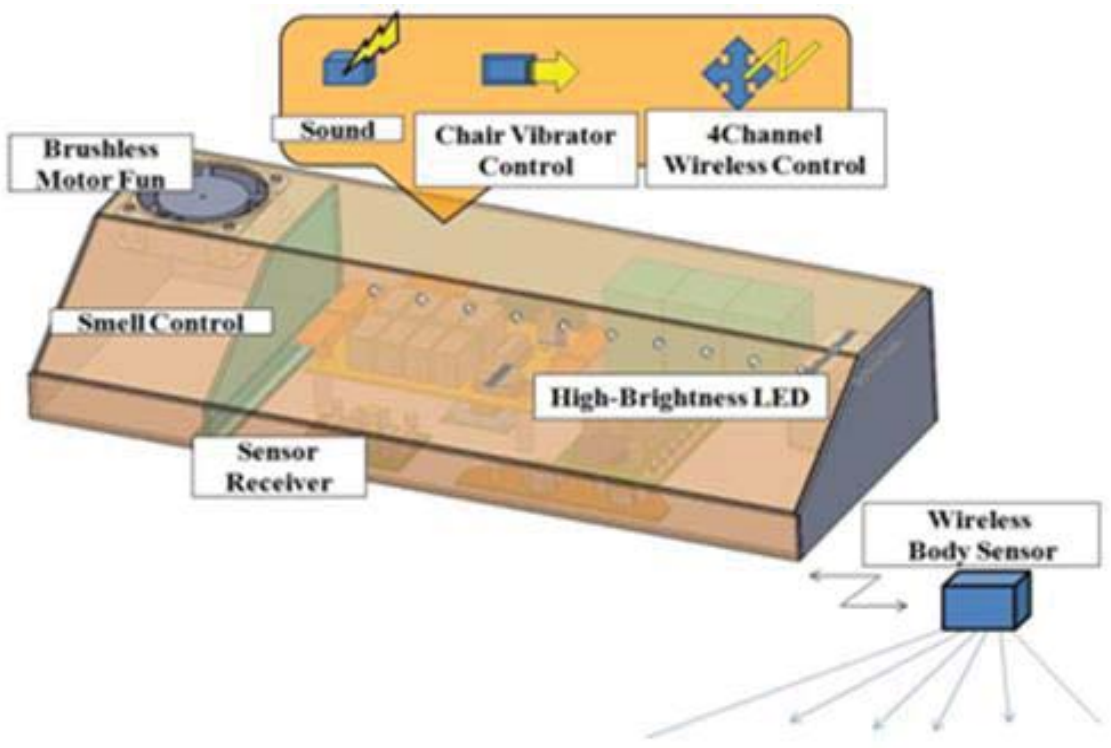

Figure 3 SmartBox functions.

These functions can calm and relax children with ASD that are anxious or on a panic attack and increase concentration on tasks. ATs are often presumed to improve health and social care services for children with autism spectrum disorders. Researchers are taking in consideration that difficulties in sustaining attention on imposed tasks may be attributable partly to a developmental delay and partly to the motivational contingencies of a task rather than to a primary impairment in the ability to sustain attention [10].

In order to keep the child motivated in learning activities we used the features of SmartBox device; the Chair Vibrator Control, Light Control, Smell Control, Sound Control.

- Chair or Bed Vibrator Control for vibrating the chair.

Through sensory integration, physical vibrations of chair will relax and calm the child.

- Light Control for adjusting the room light.

If the child is a visual learner, in order to capture child's attention we can use the computers screen to show colored images and use the light control for changing the room light. Also, if the child has sensory integration issues with different lighting we can control the setting of the lights.

- Smell Control for controlling the room smell.

If the child likes certain smells/perfumes we can put the perfume of that smell to get the attention.

- Sound Control to emit relaxing sounds.

If the child accepts auditory stimuli we can use this to get his attention and maintain focus in learning.

Tagging physical objects to find and analyze data about the object is one way the loT can be used in education. Using our proposed and implemented system, a child can learn new words through touching the physical objects that are in their vocabulary list (see Fig. 4). Each physical object would have a RFID tag placed on the item. When this tag is read by a RFID reader or scanned by an application running on a computer or mobile device it would prompt the device to open up a page of information or send a command for an action to happen.

RFID tags can be created and attached by the parents for each of the physical items in the vocabulary list. When the child places the RFID card on the RFID reader, it will say the word for the item in their native language. Touching the item will give to the child another sense to be engaged and may help them learn new words faster. 


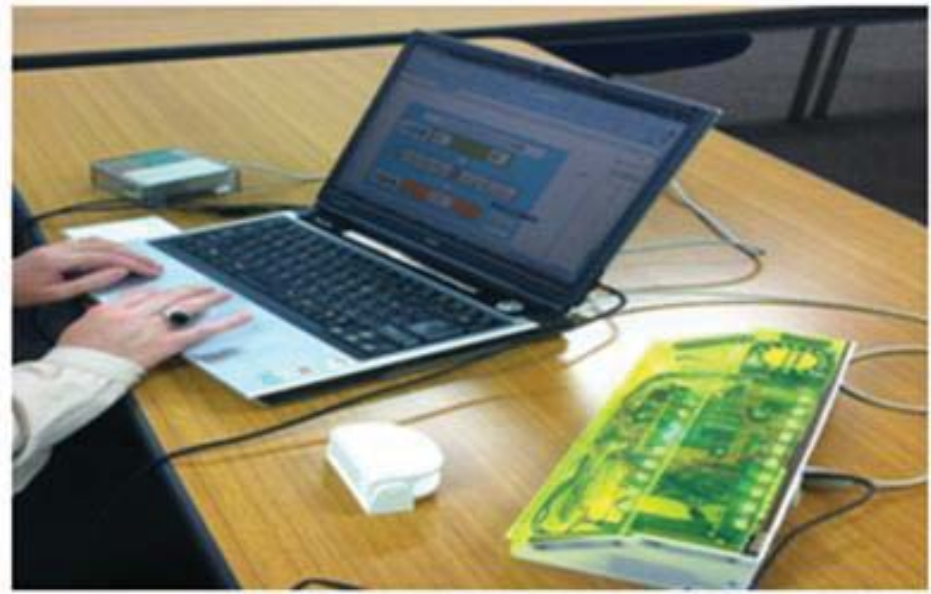

Figure 4 Proposed smart environment

Every IC Tag card can be connected with Powerpoint slides (one IC Tag for one slide). All information is saved in an Access database. Every time that IC Tag card touches the IC Tag reader, the slide connected with this card is displayed on computer screen (except computer screen also video projector, robots can be used).

Our system can generate random presentation and flash presentation (the interval of flash presentation can be set manually). IC Tag cards can be shuffled as playing cards.

The experimental results showed the following:

- The smart environment can help children with autism stay focused during their learning and can maximize their ability to reach their peak attention.

- The child's task performance is enhanced and the child will be able to learn new language skills, social skills, appropriate behavior and academic skills.

- The child's concentration and the time amount of the study are improved by the usage of SmartBox.

\section{Proposal of Using the Smart Environment in the Regional Center for Autism in Albania to Support Students during Their Learning}

In Albania, there is no publicized data on the number of students within ASD. According to the report "Analysis of the history of the development of education of children with disabilities in Albania during 1945 - 2011", the number of people registered in 2010 with one or several disabilities was 123,774 and 53.783 of them are under the category "Mental, sensory, physical" [11]. In this report, there is no category for students or people with Autism.

The Ministry of Education and Science of Albania has started collecting information on the number of students with disabilities, but there is no report published yet on the number of students within ASD. The law No. 69/2012 "For the PreUniversity Education System in the Republic of Albania" that entered in force in 2012, specifically articles 6, 19, 20, 57 , 63,65 regulate the rights to education of students with special needs. There is no University in Albania that offer programs that prepare teachers to work with students with Autism. The teacher preparation programs in higher education do not offer special education teacher programs and is not established yet the list of competencies areas for teachers working with students with ASD.

Some higher education institutions like University of Tirana or University of Shkodra "Luigj Gurakuqi" offer study programs of social workers and psychologists. In these programs there are some coursework that prepares teachers to work with special needs students.

Even, in United States the Council for Exceptional Children (CEC) did not have a set of standards for training teachers for the category of autism until 2009 [12].

It is very important that the programs that prepare teachers incorporate evidence-based practices which are instructional strategies, interventions, or teaching programs that work when working with students with ASD [13]. 
According to publicized data in United States, ASDs affect one out of every 88 children in the U.S. They occur more often among boys than girls. While autism appears to be on the rise, it's unclear whether the growing number of diagnoses shows a real increase or comes from improved detection forms.

Students with learning disabilities need extra support that goes beyond classroom settings in order to succeed in school. According to the data in the United States, secondary-school-age students with autism began receiving services as young children; parents report that, on average, youth were 4 years old when they first received disability-related services from a professional [14]. By the time they are in secondary school, according to teachers, 97 percent of students with autism receive some type of accommodation or modification, learning support, technology aid, or related service to enhance their performance in school.

It is very important that education system in Albania recognizes the needs of students with ASD and supports them with different types of accommodations and assistive technologies as all researchers in this field agree that early intervention makes a big difference in the lives of the students within this category.

\subsection{Regional Center for Autism in Albania}

Diagnosing children with autism and supporting them in their youth and providing them with some kind of intervention has been a challenged subject in Albania. The main factors are the lack of methodology of early diagnoses, the lack of team of behavioral, educational and/or medical professionals, which may include a specialized teacher, Speech Language Pathologist, Occupational Therapist and Applied Behavior Analysis specialists.

In the recent years, the Albanian Children Foundation (ACF) has been very active in raising awareness regarding autism in the country. The ACF has established the first autism support center in Albania. The Regional Center for Autism in Albania is located in Tirana and aims to support and care for children with Autism Spectrum Disorder in the region and to raise public awareness in this field.

The center offers for children birth to seven early intervention programs. Since its opening the center has played an important role on working with different national and international key actors to develop the National Autism Strategy. Currently, there is no available national data on the percentage of students with Autism Spectrum Disorder in Albania. According to the specialists of the center the assumption is that Albania is not different from other countries, and the approximate estimation given by the center is that $1 \%$ of children should be within this spectrum.

The center implements internationally known best practices in order to better diagnose children, support children during their learning in order to become independent learners. The center is planning to implement a strategy to work with older children too and work with other institutions and organizations in order to establish laws that will support children during their school years.

The center is working with the best specialists in this field and one of them Deborah Grant, received a grant from Autism Speaks to conduct a research in Albania. Her research was focused on implementation of the screening method in Albanian settings and provision of continues professional support to the centers' specialists.

One of her books "Autism in your classroom" is translated in Albanian so the specialists, teachers and parents can learn about the best practices when working with children with ASD [15].

Children with a diagnosis of autism vary greatly in their strengths/weaknesses, likes/dislikes and general personality and approach to life and therefore cannot be recommended a single teaching method that can be used for all of them. In today's market, there are several teaching materials and methods that can be used based on students' needs. Centers similar to this use designed materials and activities for ASD children based on the "trial and error" method in order to find out what approach is best [16].

Also, the method of the task presentation is very important when it comes how learning disabled children perform on academic tasks. The results from a research study showed that learning disabled children performed best on a paired associated task, especially when the method of task presentation was matched with learning disabled child's optimal response mode [17].

\subsection{Benefits of using Assistive technologies to support ASD students' learning process}

The definitions for the assistive technologies and assistive technology services are given in the Individuals with Disabilities Education Act (IDEA) in 1990. Assistive technology device means any item, piece of equipment, or product system, whether acquired commercially, off-the-shelf, modified or customized, that is used to increase, maintain, or improve functional capabilities of individuals with disabilities. Assistive technology service is any service that directly 
assists an individual with a disability in the selection, acquisition, or use of an assistive technology device.

When specialist and educators make decisions in the assistive technologies to be used to support students' learning they should consider carefully students' abilities and disabilities in these areas:

Communication, academic, motor, behavior, organization, social interactions, transitions and other concerns related to the specific situation. Also, they should evaluate carefully the environmental considerations that impact the student's participation and the tasks that the students are expected to do. Parents should be involved in this decision making process.

Knowing students' abilities and disabilities would support the team of educators, specialists and parents on making decisions on the type of assistive technologies to be used to support students' learning. The assistive technologies range from low tech to high tech and depending on the students' abilities the right technology should be chosen to support students on their learning process.

By using different types of assistive technologies according to their abilities and disabilities will support them use in their advantage their strengths and gain new skills.

The areas that the assistive technologies might be used into students' daily lives in order to support them either gaining new skills or dealing with their disabilities are: visual information processing, sensory integration, motor skills development, academics, organization skills, behavior problems, social interactions, transition.

Using assistive technologies will support students with ASD gain new academic skills, social skills and support them performing and carrying out every day's tasks.

For example, individuals with ASD typically process visual information with the greatest clarity, providing them with visual supports helps to promote that strength. Various types of technology—from "low" to "high" tech—should be infused into every aspect of their daily lives.

There are many researchers in the field that they have shown that the use of AT with children within ASD is successful as long that the evaluation team takes under consideration:

- The age of the child/student

- Diagnoses of the child

- The best learning mode of the child

- The ability to sit and use the computer

- Other tools and interventions that are being used to support the child learn new skills

A good guide for the specialists of the center when working to make decisions about the proper AT tools might be the use of the some of the tools designed by the Wisconsin Assistive Technology Initiative (WATI). The Assistive Technology Supports for Individuals with Autism Spectrum Disorder is an autism manual that is designed to guide specialist assess the students and decide on the tools and strategies that might work more effectively for the student. Specialist can use some of the tools in this manual like the Information Guide tool, the Environmental Observation Guide tool, Team decision making guide tools. Also, the manual describes many Assistive Technologies that range from low tech to high tech that have been proven useful with children with autism.

Other similar centers like the Abu Dhabi Center for Autism use the assistive technology devices like the DynaVox Maestro to support students learn new communication skills. The use of ATs in this center was very successful and the results showed that students learn new communication and language skills [18].

Based on these positive results we propose to use the smart environment combined with other proven assistive Technologies in the Albanian center in order to support students during learning process. In our previous work, we implemented and evaluated the performance of a new system based on Internet of Things (IoT) and P2P technology for supporting learning and improving the quality of life for children with ASD.

Through our proposed system, we intend to get and maintain the child's attention by obtaining the calm-alert state window in which the ability to function is maximized [19]. In this state the child's nervous system is sufficiently aroused for peak attention and task performance which enhances a person's ability to register and orient to sensory information.

Our proposed system can detect the child's movement by using hand sensor and body sensor. When a sensor reacts, the interval time will be reset to zero. From the measurement data of the child's hand movements we can consider the point of effective stimulation for the child from the sensing rate values.

The experimental results showed that the child's concentration and the time amount of the study are improved compared to the case not using the SmartBox.

The smart environment can help children with autism stay focused during their learning and can maximize their ability to reach their peak attention. The child's task performance is enhanced and the child will be able to learn new 
language skills, social skills, appropriate behavior and academic skills. The child's concentration and the time amount of the study are improved by the usage of SmartBox.

During learning activities based on our smart environment, we propose the use of visual learning aids like language builder picture cards - nouns to teach receptive language and expressive language which will enhance student's communication skills. Also, we propose to use the mathematics skills builder cards to teach numbers and basic math skills.

Some of the activities that can be used are matching identical objects, and matching similar objects which supports gaining new social skills.

We propose that educators carefully chose and combine the assistive technologies, the activities and individualized curriculum to target a specific need of the student.

\section{Conclusions and Future Work}

In this paper, we proposed the use of the smart environment based on P2P technology and loT in the Regional Center for Autism in Tirana as a form of assistive technology aiming to assist/support children with ASD getting new communication and language skills.

The use of this environment aims to increase child's concentration during learning and get and maintain his calm alert window in order to maximize the child's ability to function.

In the future we would like to use the proposed system combined with different types of high-tech assistive technologies and evaluate the experimental results. Also, we would like to use the proposed system with purpose of improving the quality of life of children with ASD and support them to lead independent lives.

\section{References}

American Psychiatric Association, "Diagnostic and Statistical Manual of Mental Disorders", 4th Edition. Washington, DC, 2000.

Autism Spectrum Disorders, http://www.webmd.com/brain/autism/autism-spectrum-disorders.

Albanian children Foundation, www.albanianchildren.org.

Tomlinson \& Allan, "Leadership for Differentiating Schools \& Classrooms", ISBN-13: 978-0-87120-502-5, 2000.

Home Networking, http://www.iec.org.

A. Tapus, M. Matarich, B. Scassellati, "The Grand Challenges in Socially Assistive Robotics", Robotics \& Automation Magazine, IEEE, vol. 14, no. 1, pp. 35-42, 2007.

Y. Huang, G. Li, "Descriptive models for Internet of Things", Proc. of Intelligent Control and Information Processing International Conference (ICICIP-2010), pp.483-486, 2010.

"Sensory Treatment Yields Promising Results for Children with Autism", http://news.temple.edu/news/sensory-treatmentyieldspromising-results-children-autism.

C. Murray-Slutsky, B. A. Paris, "Exploring the Spectrum of Autism and Pervasive Developmental Disorders", Therapy Skill Builders, 2000.

H. B. Garretson, D. Fein, L. Waterhouse, "Sustained Attention in Children with Autism", Journal of Autism and Developmental Disorders, Vol. 20, Issue 1, pp 101114, 1990.

"Analysis of the history of the development of education of children with disabilities in Albania during 1945 - 2011", World Vision Report, March 2012. http://wvi.org/albania/publication/right-inclusive-education-children-disabilities-analysis-history-educational

C. Baker, "Preparing Teachers for Students with Autism" , Special Education Journal, John Hopkins university School of Education, http://education.jhu.edu/PD/newhorizons/Journals/specialedjournal/BakerC

T. Marder, D. Fraser, "Evidence-Based Practice for Special Educators Teaching Students with Autism", Special Education Journal, John Hopkins university School of education.

Wagner, M., Newman, L., Cameto, R., Levine, P., and Marder, C. (2003). Going to School: Instructional Contexts, Programs, and Participation of Secondary School Students with Disabilities. Menlo Park, CA: SRI International.

Fein, D. and Dunn, M. (2007) Autism in Your Classroom: A Guide for Regular Education Teachers. NY: Woodbine House

Sheila Bell "Teaching Math with Meaning",http://www.autismandtheartofcommunication.com/files/Download/Teaching\%20Math\%20with $\% 20$ Meaning.pdf, January 2002

John T. Braggio, Sherryll M. Braggio, Joseph H. Lanier, Larry Simpson, Fredericka K. Riesman, "Optimal Response Modes Influence the Performance of Learning Disabled Children on Academic Tasks", Journal of Learning Disability, 1979

Abu Dhabi Center for Autism, case study, Microsoft Case Study. http://www.microsoft.com/casestudies/Case_Study _Detail.aspx?CaseStudyID=4000011348 
Anna Nguyen, "Sensory Treatment Yields Promising Results for Children with Autism", Temple Times 2008, http://news.temple.edu Inews/sensory-treatment-yields-promising-results-children-autism 